\title{
Computational Zooming in Near Unilateral Cracks by Schwarz Method with Total Overlap
}

\author{
Faker Ben Belgacem ${ }^{1}$, Nabil Gmati ${ }^{2}$ and Faten Jelassi ${ }^{1, *}$ \\ ${ }^{1}$ Alliance Sorbonne Université, UTC, EA 2222, Laboratoire de Mathématiques \\ Appliquées de Compiègne, F-60205 Compiègne, France. \\ ${ }^{2}$ College of Sciences \& Basic and Applied Scientific Research Center, \\ Imam Abdulrahman Bin Fä̈sal University, Dammam, Saudi Arabia.
}

Received January 30, 2019; Accepted April 27, 2019

\begin{abstract}
We focus on the numerical solver of unilateral cracks by the Schwarz Method with Total Overlap. The aim is to isolate the treatment at the vicinity of the cracks from other regions of the computational domain. This avoids any direct interaction between specific approximations one may use around the singularities born at the tips of the cracks and more standard methods employed away from the cracks. We apply an iterative sub-structuring technique to capture the small structures by insulating the cracks into patches and making a zoom around each of them. The macro-problem is in turn set on the whole domain. As for the classical Schwarz method, the communication between the micro (local) and macro (global) levels is achieved iteratively through some suitable boundary conditions. The micro problem is fed by Dirichlet data along the (outer) boundary of the patches. The specificity of our approach is that the macro problem inherits transmission conditions. Although they are expressed across the cracks, the final algebraic system to invert is blind to the discontinuities of the solution. In fact, the stiffness matrix turns out to be the one related to a safe domain, as if cracks were closed or the unilateral singularities were switched off. Only the right hand side is affected by what happens at the vicinity of the cracks. This enables users to run one of many efficient algorithms found in the literature to solve the linear macro-problem. In the other hand side, in spite of the still bad conditioning and the non-linearity of the unilateral micro problems, they are reduced in size and may be inverted properly by convex optimization algorithms. A successful convergence analysis of this variant of the Schwarz Method is performed after adapting to the unilateral non-linearity the variational tools developed by P. L. Lions.
\end{abstract}

AMS subject classifications: 35N86, 65N55

\footnotetext{
*Corresponding author. Email addresses: faker.ben-belgacem@utc.fr (F. Ben Belgacem), faten.jelassi@
} utc.f (F. Jelassi), nmgmati@iau.edu.sa (N. Gmati) 
Key words: Integral operators, regular kernels, Jacobi transform, separated variables approximation.

\section{Introduction}

Realistic cracks that occur in many engineering fields are most often subjected to non linear unilateral contact conditions. Designing affordable methodologies for their reliable numerical simulation still arises substantial interest. Multifold enhancements may be on increasing the accuracy of the approximation, on improving the efficiency of the solvers to run for evaluating the discrete solution or on the implementation and parallelization procedures of these solvers, ... etc.

Enrichments of finite element methods have been elaborated to strengthen the accuracy of the discrete displacement, strain and stress fields. A qualitative analysis of the singularities arisen at the vicinity of crack tips is now known and practitioners can therefore refine the mesh in a well adapted shape so to well capture the singular part of the solution. Newer procedures may also be employed to improve the computed solution (see $[5,13,14,32,33,35,37])$. We point out, in particular, different versions of X-fem, PU-fem and G-Fem with or without (Heaviside) cut-off functions (see $[2,9,10,36])$. The reverse of the medal has to do with the condition number of the discrete problems to solve. These problems suffer from bad and possibly very bad conditioning. We refer for instance to $[2,10]$ for some convincing examples. Solving efficiently the discrete system, after carrying out a finite element approximation of any kind, requires some known effective techniques such as multi-grid, multi-scale or sub-structuring approaches. This is the way followed for instance in $[7,15,24,26,38,39]$. We pursue here a similar objective by applying the iterative Schwarz Method with Total Overlap for the unilateral cracks introduced in for linear problems in $[6,23]$. The aim here is the realization, at each iteration, of a numerical zoom where it is needed, in a thin region surrounding the cracks. Zooming methods has been used in the finite elements analysis of the linear case and proved to be helpful in well capturing the localized singularities. We recommend $[3,4,7,8,11,15,16,20,22,28,29,31,34]$ and references therein.

The purpose is hence to apply the Schwarz Method with Total Overlap to compute the displacement or temperature field at the vicinity of cracks where the contact is of Signorini's type. It is successfully used and analyzed for linear cracks in [6]. It helps making a numerical zoom around the geometrical singularities, born along and especially at the tips of the cracks. The displacement field satisfies then a variational inequality within a patch surrounding the cracks. To complete the construction of the full solution, a linear problem is solved in the whole domain. Both problems talk to each other alternately through some suitable transmission conditions. How the coupling operates is the distinctive marker of the method. The core advantage is related to the linear problem which can be set up on the safe domain. Things occur as if the cracks are closed, at least for the stiffness matrix. The straightforward result is the enhancing of the condition number of 
that matrix as observed in [30]. We perform the convergence analysis in the nonlinear Signorini context. The mathematical methodology is rooted in [27] with the modifications required by the unilateral contact ; it enables us to state a strong convergence of the algorithm with respect to the natural norms.

The outline of the paper is as follows. In Section 2, we describe two examples from solid mechanical and hydrostatic where unilateral cracks may occur. They are modeling semi-permeable leaks in the latter and fatigue fractures in the former. We then write down the variational inequality for the thermal problem and conduct a brief discussion about the singularities born at the vicinity of the tips of the cracks. This suggests to practice a zoom around these special points to get a better insight on their singular behavior. Section 3 is precisely dedicated to the description of the numerical zooming method. The cracks are isolated within patches or (narrow) sub-domains where (local) variational inequalities should be handled. The temperature field in the remaining part of the domain is computed as the solution of a linear problem where boundary conditions along the cracks are obtained from the micro subproblems, already inverted. The non standard point resides in the nature of these informations which are transmission conditions. Different subproblems are updated iteratively by means of the alterning Schwarz process. We discuss some of its fundamental properties. Afterwards, we make a condensation along the boundaries of the patches surrounding the cracks and explain why our iterative algorithm is equivalent to the Picard method applied to a particular nonlinear operator we define here. In Section 4, we state and prove an abstract convergence result in a Hilbert space of the Picard sequence. In Section 5, the abstract result is applied to our Schwarz procedure which allows us to derive a convergence result of the Schwarz iterates toward the exact solution, with respect to the energy norm.

Some notations - Let $\Omega$ be a bounded domain in $\mathbb{R}^{d}, d=2,3$, with a boundary $\Gamma$. The generic points of $\Omega$ are denoted by $x$ or $y$. The Lebesgue space $L^{2}(\Omega)$ of square integrable complex valued functions is endowed with the natural norm $\|\cdot\|_{L^{2}(\Omega)}$ and we set $L^{2}(\Omega)=$ $L^{2}(\Omega)^{d}$. We need also some Sobolev spaces, $H^{1}(\Omega)$ involves all the functions that are in $L^{2}(\Omega)$ so as their partial derivatives. The subspace containing the functions in $H^{1}(\Omega)$ that vanish on $\Gamma$ is denoted by $H_{0}^{1}(\Omega)$. The set of the traces over $\Gamma$ of all the functions of $H^{1}(\Omega)$ is denoted $H^{1 / 2}(\Gamma)$ and $H^{-1 / 2}(\Gamma)$ is its dual (see [1]). The symbol [·] stands for the jump across a given boundary.

\section{Nonlinear cracks}

Cracks initiation or cracks growth that currently occur in mechanical or thermal structures due to fatigue are non linear phenomena. Unilateral conditions or the Signorini type conditions versus linear Dirichlet or Neumann boundary conditions are generally adopted along those cracks. Many examples involving nonlinear crack models may be presented. We choose to briefly describe two configuration, one picked-up in mechanical structures and the other in thermal diffusion. 


\subsection{Fatigue cracks in the elasticity model}

Consider an elastic solid that occupies the domains $\Omega \subset \mathbb{R}^{d}$ in its unconstrained configuration. The boundary is $\partial \Omega$ and the unit external normal is denoted by $\boldsymbol{n}$. We assume that $\partial \Omega$ is partitioned into $\Gamma_{D}$ and $\Gamma_{N}$. We suppose that $\Omega$ contains a crack represented by a connected Lipschitz surface $\gamma$, entirely embedded in $\Omega\left({ }^{+}\right)$. We denote $\Omega_{\gamma}=\Omega \backslash \gamma$ (see Figure 1). To specify the boundary conditions on the crack $\gamma$, we need the symbols $\gamma^{+}$ and $\gamma^{-}$to distinguish both lips of $\gamma$. We denote by $\boldsymbol{n}$ the unit normal to $\gamma$ oriented from $\gamma^{+}$toward $\gamma^{-}$.

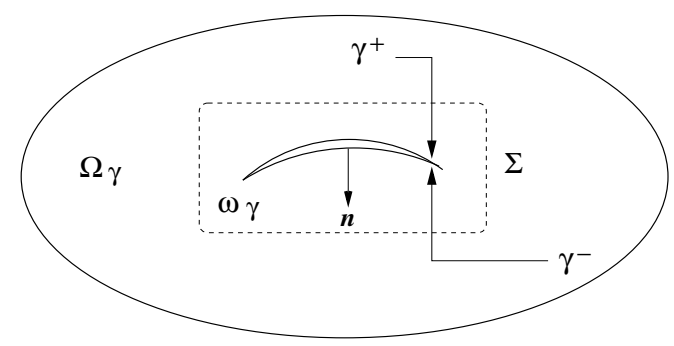

Figure 1: The cracked solid. Inter-penetration is not authorized along the crack $\gamma$. The surface $\Sigma$ is only indicative.

The solid $\Omega$ is fixed along $\Gamma_{D}$ and is subjected to a surface solicitation $g \in L^{2}\left(\Gamma_{N}\right)$ on $\Gamma_{N}$ and to a volume force $f \in L^{2}(\Omega)$, which is most often its own weight. The elastic system we are concerned with consists in: finding a displacement fields $u$ satisfying the boundary value equation

$$
\begin{array}{ll}
-\boldsymbol{d i v} \sigma(\boldsymbol{u})=\boldsymbol{f} & \text { in } \Omega_{\gamma}, \\
\boldsymbol{u}=0 & \text { on } \Gamma_{D}, \\
\sigma(\boldsymbol{u}) \boldsymbol{n}=\boldsymbol{g} & \text { on } \Gamma_{N} .
\end{array}
$$

The symbol div stands for the divergence operator of a tensor field and is defined by $\operatorname{div} \sigma=\left(\frac{\partial \sigma_{k r}}{\partial x_{r}}\right)^{k}$. The body is made of Hook material whose constitutive law is provided by $\sigma(\boldsymbol{u})=A(\boldsymbol{x}) \varepsilon(\boldsymbol{u})$, where $A(\boldsymbol{x})$ is a fourth order symmetric and elliptic tensor. Frictionless unilateral contact conditions are enforced on $\gamma$ required to close the system,

$$
\begin{aligned}
& {[\boldsymbol{u} \cdot \boldsymbol{n}] \leq 0, \quad[(\boldsymbol{s} \boldsymbol{n}) \cdot \boldsymbol{n}]=0, \quad \boldsymbol{s}_{\boldsymbol{n}} \geq 0, \quad\left\langle\boldsymbol{s}_{\boldsymbol{n}},[\boldsymbol{u} \cdot \boldsymbol{n}]\right\rangle=0,} \\
& \boldsymbol{s}_{\tau}^{+}=\boldsymbol{s}_{\tau}^{-}=0 .
\end{aligned}
$$

The notation $[\boldsymbol{u} \cdot \boldsymbol{n}]=\left(\boldsymbol{u}^{+} \cdot \boldsymbol{n}-\boldsymbol{u}^{-} \cdot \boldsymbol{n}\right)$ stands for the jump of the normal displacements across the contact zone $\gamma$ and $s_{n}=(s \boldsymbol{n}) \cdot \boldsymbol{n}$ is the normal stress while $\sigma_{\tau}=\sigma-\boldsymbol{s}_{n} \boldsymbol{n}$ is the tangential stress. Condition (2.5) indicates that the contact occurs without friction while (2.4) are

\footnotetext{
${ }^{\dagger}$ The assumption that $\gamma$ is connected does not restrict the generality.
} 
the Signorini conditions, they are non-linear and involve no-penetration inequalities, notensile-contact-stresses inequalities and complementarity relation.

\subsection{Thermal unilateral cracks}

Another problem where unilateral cracks may be modeled by a variational inequality pertains to the thermostatic or hydrostatic problem. A different configuration, from the physical view, may yield to a similar model of Signorini unilateral conditions. An example may be provided by a thermal reservoir or a water tank, based on the principle of storing heat or water, for a later reuse. Consider the quasi-static thermal problem exposed in [12]. In the water reservoir the physical quantity to look at is the pressure. To fix the ideas, we restrict ourselves to the thermal reservoir, and we are then interested in the temperature field $\varphi$ in a heat conducting domain $\Omega$. Let $\gamma$ be an internal heating leak surface causing thermal discontinuity. One lip of $\gamma$ is denoted $\gamma^{+}$and the other is $\gamma^{-}$. As previously, we set $\Omega_{\gamma}=\Omega \backslash \gamma$. Assume given $g \in L^{2}(\Gamma)$ and $f \in L^{2}(\Omega)$. The heat problem consists in finding $(\varphi)$, satisfying the following equations:

$$
\begin{array}{ll}
\varphi-\operatorname{div}(c \nabla \varphi)=f & \text { in } \Omega_{\gamma}, \\
\left(c \partial_{n}\right) \varphi=g & \text { on } \Gamma .
\end{array}
$$

The heat conductivity of the media $c$ is generally piecewise regular, belongs to $L^{\infty}(\Omega)$ and is bounded away from zero. To complete and close the model, we provide unilateral conditions along $\gamma$

$$
\begin{array}{ll}
{[\varphi]=\varphi^{+}-\varphi^{-} \geq 0,} & {\left[c \partial_{n} \varphi\right]=0,} \\
\left(c \partial_{n}\right) \varphi \geq 0, & \left\langle\left(c \partial_{n}\right) \varphi,[\varphi]\right\rangle=0 .
\end{array}
$$

The variational setting - Given that the cracked domain $\Omega_{\gamma}$ is an open domain, the construction of the Sobolev space $H^{1}\left(\Omega_{\gamma}\right)$ is defined as in [17]. The functions in $H^{1}\left(\Omega_{\gamma}\right)$ may have a jump across $\gamma$. That jump $[\psi]$ belongs to $H_{00}^{1 / 2}(\gamma)$ (see [1]). The notation $\langle\cdot, \cdot\rangle$ in the complementary condition is the duality between $H_{00}^{1 / 2}(\gamma)$ and $\left(H_{00}^{1 / 2}(\gamma)\right)^{\prime}$. Throughout the paper we use the following norm

$$
\|\psi\|_{H^{1}\left(\Omega_{\gamma}\right)}^{2}=\|\psi\|_{L^{2}\left(\Omega_{\gamma}\right)}^{2}+\|\sqrt{c} \nabla \psi\|_{L^{2}\left(\Omega_{\gamma}\right)}^{2} .
$$

Taking into account the unilateral contact condition on $\gamma$ in the weak formulation is made by incorporating it in the admissible closed convex cone

$$
K(\Omega)=\left\{\psi \in H^{1}\left(\Omega_{\gamma}\right), \quad([\psi])_{\mid \gamma} \geq 0\right\} .
$$

We apply the variational principle for the semi-permeable tank to produce the variational inequality consisting in : finding $\varphi \in K(\Omega)$ such that:

$$
\int_{\Omega}[\varphi(\psi-\varphi)+c \nabla \varphi \nabla(\psi-\varphi)] d x \geq \int_{\Omega} f(\psi-\varphi) d x+\int_{\Gamma} g(\psi-\varphi) d \Gamma, \quad \forall \psi \in K(\Omega) .
$$


Using here again Stampacchia theorem we state the well-posedness of the variational inequality (2.10) which has therefore only one solution $\varphi \in K(\Omega)$ that depends continuously on the data $(f, g)$ (see $[19,25])$.

\section{Schwarz algorithm with a total overlap}

Now, we turn to the description of the Schwarz algorithm applied to the Laplace-Signorini problem. Let us encircle the crack $\gamma$ by a closed surface $\Sigma$ in $\Omega_{\gamma}$ and denote by $\omega_{\gamma}$ the internal domain delimited by $\Sigma$ and containing $\gamma$ (see Figure 2). The iterative method we aim at is based on an exchange of some boundary conditions between the two domains $\omega_{\gamma}$ and $\Omega_{\gamma}$ that turns to be profitable.

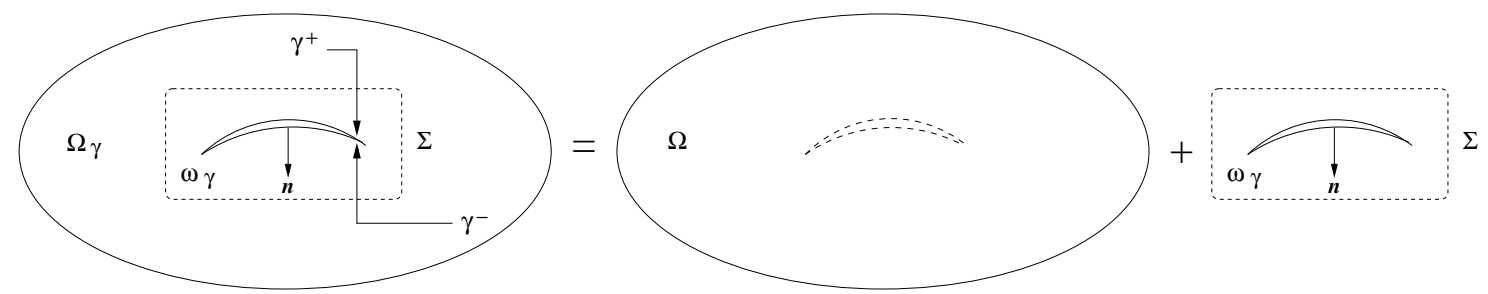

Figure 2: Global-local sub-structuring scheme. Dashed lines for the crack are to say that the discrete problem to solve does not really see the crack.

The starting point of the total overlapping Schwarz Algorithm is to replace (2.6)-(2.8) by two coupled subproblems. One, set on the whole domain $\Omega_{\gamma}$, is a linear transmission problem that consists in: finding $\eta \in H^{1}\left(\Omega_{\gamma}\right)$ such that

$$
\begin{array}{ll}
\eta-\operatorname{div}(c \nabla \eta)=f & \text { in } \Omega_{\gamma,} \\
{[\eta]=[\chi]} & \text { on } \gamma, \\
{\left[\left(c \partial_{n}\right) \eta\right]=0} & \text { on } \gamma, \\
\left(c \partial_{n}\right) \eta=g & \text { on } \Gamma .
\end{array}
$$

The other is set on $\omega_{\gamma}$, is nonlinear and involved with the unilateral contact conditions along the crack. It is written as : find $\chi \in H^{1}\left(\omega_{\gamma}\right)$ such that

$$
\begin{array}{lll}
\chi-\operatorname{div}(c \nabla \chi)=f & \text { in } \omega_{\gamma}, \\
\chi=\eta & \text { on } \Sigma, \\
{[\chi] \geq 0, \quad\left[c \partial_{n} \chi\right]=0,} & \left(c \partial_{n}\right) \chi \geq 0, \quad\left\langle\left(c \partial_{n}\right) \chi,[\chi]\right\rangle=0 & \text { on } \gamma .
\end{array}
$$

Remark 3.1. The idea we pursue through this decomposition is the possibility to carry out two independent computations with different resolutions, when both sub-problems are uncoupled by an iterative process. An accurate method is recommended in the local sub-domain $\omega_{\gamma}$ to simulate the small scales at the vicinity of the crack, either by constructing a dense mesh or by using an $X$-fem $G$-fem or $P U$-fem approximations (see $[10,32])$. 
We practice then a numerical zoom. The method to compute $\eta$ in the global domain $\Omega$, which contains bigger scales far away from the cracks is rather of a moderate resolution. Moreover, the problem to solve is linear. Both facts may therefore spare a substantial computational time and costs.

Priori to the application of an iterative algorithm so to break the coupling between the transmission sub-problem (3.1)-(3.4) and the reduced unilateral sub-problem (3.5)-(3.7), we prove the following result holds.

Lemma 3.1. There is a unique solution $(\eta, \chi) \in H^{1}\left(\Omega_{\gamma}\right) \times H^{1}\left(\omega_{\gamma}\right)$ to the coupled problems (3.1)(3.4) and (3.5)-(3.7). Moreover, we have that $(\eta, \chi)=\left(\varphi, \varphi_{\mid \omega_{\gamma}}\right)$.

Proof. Straightforwardly $\left(\varphi, \varphi_{\mid \omega_{\gamma}}\right)$ is solution. For the uniqueness, it is easy seen that $(\chi-\eta)$ satisfies the boundary value problem

$$
\begin{array}{ll}
(\chi-\eta)-\operatorname{div}(c \nabla(\chi-\eta))=0 & \text { in } \omega_{\gamma}, \\
{[(\chi-\eta)]=0} & \text { on } \gamma, \\
{\left[\left(c \partial_{n}\right)(\chi-\eta)\right]=0} & \text { on } \gamma, \\
(\chi-\eta)=0 ? & \text { on } \Sigma .
\end{array}
$$

We deduce thus that $\chi=\eta$ (in $\omega_{\gamma}$ ) and eventually $\eta=\varphi$ (in $\Omega_{\gamma}$ ) and $\chi=\varphi$ (in $\omega_{\gamma}$ ). The proof is complete.

\subsection{The iterative scheme}

The iterative method we are interested in has been addressed for the linear cracks in [6] and its efficiency is fully settled in both theoretical and computational grounds. We operate an uncoupling on both subproblems by means of an iterative process. Assume $\left(\chi^{m}, \eta^{m}\right)$ are known, $\eta^{m+1} \in H^{1}\left(\Omega_{\gamma}\right)$ is the solution of the linear global problem

$$
\begin{array}{ll}
\eta^{m+1}-\operatorname{div}\left(c \nabla \eta^{m+1}\right)=f & \text { in } \Omega_{\gamma,} \\
{\left[\eta^{m+1}\right]=\left[\chi^{m}\right]} & \text { on } \gamma, \\
{\left[\left(c \partial_{n}\right) \eta^{m+1}\right]=0} & \text { on } \gamma, \\
\left(c \partial_{n}\right) \eta^{m+1}=g & \text { on } \Gamma,
\end{array}
$$

and $\chi^{m+1} \in H^{1}\left(\omega_{\gamma}\right)$ satisfies the nonlinear restricted problem

$$
\begin{aligned}
& \chi^{m+1}-\operatorname{div}\left(c \nabla \chi^{m+1}\right)=f \quad \text { in } \omega_{\gamma}, \\
& \chi^{m+1}=\eta^{m+1} \quad \text { on } \Sigma \text {, } \\
& {\left[\chi^{m+1}\right] \geq 0, \quad\left[\left(c \partial_{n}\right) \chi^{m+1}\right]=0, \quad\left(c \partial_{n}\right) \chi^{m+1} \geq 0,} \\
& \left\langle\left(c \partial_{n}\right) \chi^{m+1},\left[\chi^{m+1}\right]\right\rangle=0, \quad \text { on } \gamma \text {. }
\end{aligned}
$$

The recurrence makes a mathematical sense, and results in two coherent sequences $\left(\chi^{m}\right)_{m} \subset$ $H^{1}\left(\omega_{\gamma}\right)$ and $\left(\eta^{m}\right)_{m} \subset H^{1}\left(\Omega_{\gamma}\right)$. 
The iterative algorithm presented here may be connected to a particular class of Schwarz method, well formalized in [6], with a total overlap given that $\omega_{\gamma}$ is entirely included in $\Omega_{\gamma}$. The fundamental novelty resides on the way both sub-problems talk to each other. The conditions inherited by $\eta^{m+1}$ from $\chi^{m}$ are of transmission type rather than pure Dirichlet or Neumann conditions which are used for the classical version of Schwarz methods. The distinctive improvement specifically linked to these transmission conditions is that when the macro-problem (3.8)-(3.11) is discretized, the related stiffness matrix is blind to the cracks. Things happen as if the cracks are perfectly closed. Of course, they have an effect on the right hand side of the algebraic problem to cope with. In the opposite, when Dirichlet or Neumann data are selected, the cracks have a important influence on the stiffness matrix and may affect tremendously its condition number.

Remark 3.2. When the domain $\Omega_{\gamma}$ contains a finite number of connected cracks, the subdomain $\omega_{\gamma}$ may be multiply connected. Each connected component encloses a single crack. The Signorini problems are disconnected.

Remark 3.3. Antipode cases of a fully open crack or a fully shut one are linear. For the first, the active boundary condition is $\left(c \partial_{n}\right) \chi=0$ on both lips $\gamma^{+}$and $\gamma^{-}$. In the second, we have that $[\chi]=0$ and $\left[c \partial_{n} \chi\right]=0$ and things happen as if no crack existed. The total Schwarz algorithm in these two configurations have been addressed, in [6] for the former where a linear convergence is proved and for the latter the convergence is ensured in a single iteration. The in-between case turns to be nonlinear which complicates the analysis.

Problem (3.8)-(3.11) may be changed into another completely disconnected from the cracks. Indeed, we may consider an intermediary surface $\gamma_{*}$, between $\gamma$ and $\Sigma$, that in general follows the edges of the finite element mesh used for the discretization of $\eta$. Then, we write down the following problem: find $\eta_{*}^{m+1}$ such that

$$
\begin{array}{ll}
\eta_{*}^{m+1}-\operatorname{div}\left(c \nabla \eta_{*}^{m+1}\right)=f_{*} & \text { in } \Omega_{\gamma_{*}} \\
{\left[\eta_{*}^{m+1}\right]=\chi^{m}} & \text { on } \gamma_{* \prime} \\
{\left[\left(c \partial_{n}\right) \eta_{*}^{m+1}\right]=\left(c \partial_{n}\right) \chi^{m}} & \text { on } \gamma_{* \prime} \\
\left(c \partial_{n}\right) \eta_{*}^{m+1}=g & \text { on } \Gamma .
\end{array}
$$

$f_{*}$ is the extension of $f$ by zero in the sub-region of $\Omega$ inside $\gamma_{*}$.

Notice that the introduction of such $\gamma_{*}$ to hide the cracks may have an interesting impact in the implementation when finite elements are used. In addition, the iterative algorithm is not influenced by this modification. Indeed, the following lemma holds.

Lemma 3.2. The sequence $\left(\chi^{m}\right)_{m}$ defined by the total overlapping Schwarz algorithm (3.15)-(3.18) and (3.12)-(3.14) is independent of $\gamma_{*}$.

Proof. The statement holds true if we prove that the Dirichlet condition (3.13) on $\chi^{m+1}$ does not change which means that the equality to $\eta_{*}^{m+1}=\eta^{m+1}$ holds on $\gamma^{*}$. Let first 


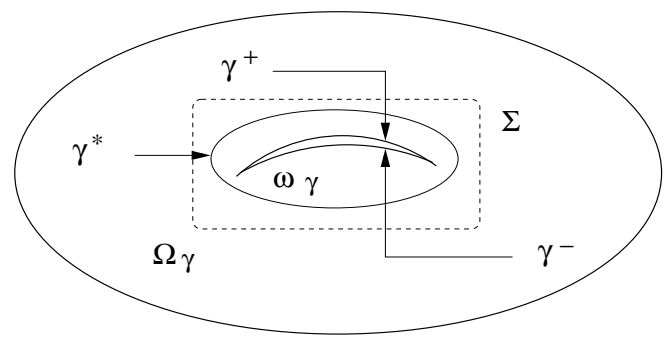

Figure 3: The transmission conditions may be written on $\gamma^{*}$ instead of $\gamma$.

denote by $\omega_{\gamma}^{*}$ the sub-domain encircled by $\gamma_{*}$ and surrounding the crack $\gamma$. Then, we define $\tilde{\eta}^{m+1}$ as follows

$$
\tilde{\eta}^{m+1}= \begin{cases}\eta_{*}^{m+1}+\chi_{m} & \text { in } \omega_{\gamma^{\prime}}^{*} \\ \eta_{*}^{m+1} & \text { in } \Omega \backslash \omega_{\gamma}^{*} .\end{cases}
$$

It is direct that

$$
\tilde{\eta}^{m+1}-\operatorname{div}\left(c \nabla \tilde{\eta}^{m+1}\right)=f, \quad \text { in } \omega_{\gamma}^{*} \cup\left(\Omega \backslash \omega_{\gamma}^{*}\right) .
$$

Now, observe that owing to conditions (3.16) and (3.17) we have that

$$
\left(\left[\tilde{\eta}^{m+1}\right]\right)_{\mid \gamma_{*}}=\left(\chi^{m}-\chi^{m}\right)_{\mid \gamma_{*}}=0, \quad\left(\left[c \partial_{n} \tilde{\eta}^{m+1}\right]\right)_{\mid \gamma_{*}}=\left(c \partial_{n} \chi^{m}-c \partial_{n} \chi^{m}\right)_{\mid \gamma_{*}}=0,
$$

this allows to deduce that

$$
\tilde{\eta}^{m+1}-\operatorname{div}\left(c \nabla \tilde{\eta}^{m+1}\right)=f, \quad \text { in } \Omega_{\gamma} .
$$

Furthermore, it is easily seen that

$$
\left(\left[\tilde{\eta}^{m+1}\right]\right)_{\mid \gamma}=\left(\left[\chi^{m}\right]\right)_{\mid \gamma}, \quad\left(\left[\left(c \partial_{n}\right) \tilde{\eta}^{m+1}\right]\right)_{\mid \gamma}=0 .
$$

Hence, $\tilde{\eta}^{m+1}$ and $\eta^{m+1}$ are solutions to the same problem (3.8)-(3.11) which is uniquely solved. This concludes to the equality $\tilde{\eta}^{m+1}=\eta^{m+1}$. As a result, we obtain in particular that $\tilde{\eta}^{m+1}=\eta^{m+1}$ on $\gamma_{*}$. The proof is complete.

\subsection{Condensation and Picard's iterates}

On the way of the convergence analysis we have in mind a slight modification is added to the sub-problems (3.1)-(3.4) and (3.5)-(3.7). We realize a shift that brings about more ease in the theoretical study. Let therefore consider $\tilde{\varphi} \in H^{1}\left(\Omega_{\gamma}\right)$ the solution of

$$
\begin{array}{ll}
\tilde{\varphi}-\operatorname{div}(c \nabla \tilde{\varphi})=f & \text { in } \Omega_{\gamma,} \\
\left(c \partial_{n}\right) \tilde{\varphi}=0 & \text { on } \gamma^{+} \cup \gamma^{-}, \\
\left(c \partial_{n}\right) \tilde{\varphi}=g & \text { on } \Gamma .
\end{array}
$$


Then, we operate the changing on the unknowns, we consider $\eta:=(\eta-\tilde{\varphi})$ and $\chi:=(\chi-$ $\tilde{\varphi})\left({ }^{\ddagger}\right)$ instead of the original ones. Setting $\zeta=-([\tilde{\varphi}])_{\gamma} \in H_{00}^{1 / 2}(\gamma)$ and plugging all in (3.1)(3.4) and (3.5)-(3.7) we obtain that $\eta \in H^{1}\left(\Omega_{\gamma}\right)$ is such that

$$
\begin{array}{ll}
\eta-\operatorname{div}(c \nabla \eta)=0 & \text { in } \Omega_{\gamma,} \\
{[\eta]=[\chi]} & \text { on } \gamma, \\
{\left[\left(c \partial_{n}\right) \eta\right]=0} & \text { on } \gamma, \\
\left(c \partial_{n}\right) \eta=0 & \text { on } \Gamma,
\end{array}
$$

and: Find $\chi \in H^{1}\left(\omega_{\gamma}\right)$ such that

$$
\begin{aligned}
& \chi-\operatorname{div}(c \nabla \chi)=0 \quad \text { in } \omega_{\gamma}, \\
& \chi=\eta \quad \text { on } \Sigma \text {, } \\
& {[\chi] \geq \zeta, \quad\left[c \partial_{n} \chi\right]=0, \quad\left(c \partial_{n}\right) \chi \geq 0, \quad\left\langle\left(c \partial_{n}\right) \chi,[\chi]-\zeta\right\rangle=0 \quad \text { on } \gamma \text {. }}
\end{aligned}
$$

Notice that the case that $\zeta \leq 0$ corresponds to a fully open crack and then $\chi=\eta=0$, and $\tilde{\varphi}$ exactly coincides with $\varphi$. The artifice used here is only for the theoretical requirements and is by no means followed in the practice where rather the Schwarz scheme as formulated in (3.1)-(3.4) and (3.5)-(3.7) is employed.

We pursue now a static condensation of the problem on the boundary $\Sigma$ and consider the $\lambda=\chi_{\mid \Sigma} \in H^{1 / 2}(\Sigma)$ as the main unknown. We denote by (3.23)-(3.25) where instead of (3.24) we impose $\chi_{\lambda}=\lambda$ on $\Sigma$. In the same spirit, we call $\eta_{\lambda} \in H^{1}\left(\Omega_{\gamma}\right)$ the solution of (3.19)-(3.22) where (3.20) is replaced by $\left[\eta_{\lambda}\right]=\left[\chi_{\lambda}\right]$ on $\gamma$. The trace function $\lambda$ we look for, the one which allows the reconstruction of $\varphi$ is so that $\left(\eta_{\lambda}, \chi_{\lambda}\right)$ satisfies the coupled problems (3.19)-(3.22) and (3.23)-(3.25). It is therefore solution of the nonlinear fixed point problem

$$
\lambda=T_{\Sigma}(\lambda), \quad \text { in } H^{1 / 2}(\Sigma) .
$$

The nonlinear operator $T_{\Sigma}$ maps $H^{1 / 2}(\Sigma)$ into $H^{1 / 2}(\Sigma)$ and is defined by

$$
T_{\Sigma}(\mu)=\left(\eta_{\mu}\right)_{\mid \Sigma}, \quad \forall \mu \in H^{1 / 2}(\Sigma) .
$$

Because of the uniqueness of the solution $(\chi, \eta)$ of the coupled problem (3.8)-(3.11) and (3.12)-(3.14) established in Lemma 3.2, $T_{\Sigma}$ has a unique fixed point $\lambda$ that coincides with $(\varphi-\tilde{\varphi})_{\mid \Sigma}$. Observe that $T_{\Sigma}$ depends on $\zeta$, by then on $\tilde{\varphi}$ and consequently on $(f, g)$. Now, consider the Picard iterates

$$
\lambda^{m+1}=T_{\Sigma}\left(\lambda^{m}\right) \text {. }
$$

It can be checked through some easy manipulations that this is but another way to express the iterative Schwarz algorithm with the following relation $\left(\eta^{m}, \chi^{m}\right)=\left(\eta_{\lambda^{m}} \chi_{\lambda^{m}}\right)+$ $\left(\tilde{\varphi}, \tilde{\varphi}_{\mid \omega_{\gamma}}\right)$. The convergence of the algorithm is narrowly connected to the properties of $T_{\Sigma}$ and relies on the theory we propose here below for an abstract nonlinear fixed point problem.

ҒFor commodity of the presentation we accept here the notation abuses. 


\section{An abstract convergence result}

Let $(H,\|\cdot\|)$ be a Hilbert space and $T$ a nonlinear operator in $H$. For a given $\mu \in H$, we define the Picard iterates $\left(\mu_{m}\right)_{m}$ by the induction

$$
\mu_{m+1}=T \mu_{m}, \quad \forall m \in \mathbb{N} .
$$

$T$ is said to be completely-continuous if it is continuous and compact. It is asymptotically regular if for any $\mu \in H$, we have that $\left(\mu_{m+1}-\mu_{m}\right)_{m}$ converges to zero. This means that

$$
\lim _{m \rightarrow \infty}\left\|T^{m+1} \mu-T^{m} \mu\right\|=0, \quad \forall \mu \in H .
$$

We have the following convergence of the Picard sequence.

Proposition 4.1. Assume that $T$ completely-continuous, asymptotically regular and has a unique fixed point $\lambda$. Let $\left(\mu_{m}\right)_{m \in \mathbb{N}}$ be a Picard sequence. If $\left(\mu_{m}\right)_{m \in \mathbb{N}}$ is bounded then it converges towards $\lambda$.

Proof. The aim is to state that the sequence $\left(\mu_{m}\right)_{m \in \mathbb{N}}$ has $\lambda$ a the unique cluster point. On account of the compactness of $T$ and to $\left(T \mu_{m}\right)_{m \in \mathbb{N}}=\left(\mu_{m}\right)_{m \geq 1}$, that $\left(\mu_{m}\right)_{m \in \mathbb{N}}$ is bounded yields that it is pre-compact. Hence, there exists a sub-sequence $\left(\mu_{m_{k}}\right)_{k}$ that converges let us say, towards $\varrho \in H$. Due to the asymptotic regularity of $T$, we have that $\left(\mu_{m_{k}+1}=T \mu_{m_{k}}\right)_{k}$ has the same limit $\varrho$. Now, seen $T$ is continuous we have necessarily that $T \varrho=\varrho$. The uniqueness of the fixed point of $T$ yields that $\varrho=\lambda$ and $\lambda$ is a cluster point of $\left(\mu_{m}\right)_{m \in \mathbb{N}}$ and it is unique. The sequence $\left(\mu_{m}\right)_{m \in \mathbb{N}}$ converges toward $\lambda$ and the proof is complete.

\section{Convergence of the Schwarz algorithm}

The convergence of the Schwarz method is the aim here. The foundation of the analysis are appropriate variational tools applied to the linear problem on $\eta$ and to the nonlinear problem on $\chi$. In a way, the proof follows [27], the inspring reference on the subjet.

\subsection{The iterating operator}

The properties of $T_{\Sigma}$ we need to state are that it is a Lipschitz operator, asymptotically regular and compact. There holds that

Lemma 5.1. There exists a constant $\beta>0$ such that

$$
\left\|T_{\Sigma} \lambda-T_{\Sigma} \mu\right\|_{H^{1 / 2}(\Sigma)} \leq \beta\|\lambda-\mu\|_{H^{1 / 2}(\Sigma)}, \quad \forall \lambda, \mu \in H^{1 / 2}(\Sigma) .
$$

The operator $T_{\Sigma}$ is then Lipschitz. It is also compact. 
Proof. The operator $T_{\Sigma}$ may be viewed as the composition of two operators, one is defined by $R_{\Sigma}(\mu)=\left(\left[\chi_{\mu}\right]\right)_{\mid \gamma}$ and the other is determined by $S_{\Sigma}\left(\left(\left[\chi_{\mu}\right]\right)_{\mid \gamma}\right)=\left(\eta_{\mu}\right)_{\mid \Sigma}$. The first $R_{\Sigma}$ maps $H^{1 / 2}(\Sigma)$ into $H_{00}^{1 / 2}(\gamma)$ is nonlinear while the second $S_{\Sigma}$ maps $H_{00}^{1 / 2}(\gamma)$ into $H^{1 / 2}(\Sigma)$ and is linear. That $T_{\Sigma}$ is Lipschitz comes from the two facts, that $R_{\Sigma}$ is Lipschitzian and $S_{\Sigma}$ is bounded. Thought, $R_{\Sigma}$ is well known to be Lipschitzian, we propose the proof of it to be self contained. Let $\lambda \in H^{1 / 2}(\Sigma)$ be given and define $\xi_{\lambda} \in H^{1}\left(\omega_{\gamma}\right)$ to be a stable expansion of $\lambda$ that vanishes at the vicinity of $\gamma$. Decomposing $\chi_{\lambda}=\tilde{\chi}_{\lambda}+\xi_{\lambda}$, it comes out that $\tilde{\chi}_{\lambda}$ belongs to the closed convex set

$$
K_{\zeta, 0}\left(\omega_{\gamma}\right)=\left\{\psi \in H^{1}\left(\omega_{\gamma}\right), \quad([\psi])_{\mid \gamma} \geq \zeta, \quad \psi_{\mid \Sigma}=0\right\} .
$$

Moreover, writing that $\chi_{\lambda}$ is solution of (3.23)-(3.25) yields the following variational inequality: $\tilde{\chi}_{\lambda} \in K_{\zeta, 0}\left(\omega_{\gamma}\right)$ is such that

$$
\int_{\omega_{\gamma}}\left[\left(\tilde{\chi}_{\lambda}+\xi_{\lambda}\right)\left(\psi-\tilde{\chi}_{\lambda}\right)+c \nabla\left(\tilde{\chi}_{\lambda}+\xi_{\lambda}\right) \nabla\left(\psi-\tilde{\chi}_{\lambda}\right)\right] d x \geq 0, \quad \forall \psi \in K_{\zeta, 0}\left(\omega_{\gamma}\right) .
$$

As a result, $\tilde{\chi}_{\lambda}$ is the projection of $\left(-\xi_{\lambda}\right)$ on $K_{\zeta, 0}\left(\omega_{\gamma}\right)$. We deduce that

$$
\left\|\tilde{\chi}_{\lambda}-\tilde{\chi}_{\mu}\right\|_{H^{1}\left(\omega_{\gamma}\right)} \leq\left\|\xi_{\lambda}-\xi_{\mu}\right\|_{H^{1}\left(\omega_{\gamma}\right)} .
$$

Calling for the trace theorem and the stability of $\left(\xi_{\lambda}, \xi_{\mu}\right)$ with respect to $(\lambda, \mu)$, we end to

$$
\left\|\left[\chi_{\lambda}-\chi_{\mu}\right]\right\|_{H_{00}^{1 / 2}(\gamma)} \leq \beta^{\prime}\left\|\chi_{\lambda}-\chi_{\mu}\right\|_{H^{1}\left(\omega_{\gamma}\right)} \leq 2 \beta^{\prime}\left\|\xi_{\lambda}-\xi_{\mu}\right\|_{H^{1}\left(\omega_{\gamma}\right)} \leq \beta^{\prime \prime}\|\lambda-\mu\|_{H^{1 / 2}(\Sigma)},
$$

which proves the first result. The constant $\beta^{\prime \prime}$ depends of course on $\zeta$. Now, the compactness of $T_{\Sigma}$ holds true if $S_{\Sigma}$ is compact. This is ensued from the elliptic regularity of the transmission problem (see [17]). Indeed, we have that actually $\eta_{\lambda} \in H^{1 / 2+\epsilon}(\Sigma)$ for some $\epsilon>0$ with the stability

$$
\left\|S_{\Sigma}\left(\left[\chi_{\mu}\right]\right)\right\|_{H^{1 / 2+\varepsilon}(\Sigma)}=\left\|\eta_{\lambda}\right\|_{H^{1 / 2+\varepsilon}(\Sigma)} \leq \beta^{\prime \prime \prime}\left\|\left[\chi_{\lambda}\right]\right\|_{H_{00}^{1 / 2}(\gamma)} .
$$

The compactness of $S_{\Sigma}$ is achieved owing to the compactness of the embedding of $H^{1 / 2+\epsilon}$ $(\Sigma)$ into $H^{1 / 2}(\Sigma)$. The proof is complete.

Lemma 5.2. The operator $T_{\Sigma}$ is asymptotically regular. Moreover, for any $\mu \in H^{1 / 2}(\Sigma)$ the sequence $\left(\left(T_{\Sigma}\right)^{m} \mu\right)_{m}$ is bounded in $H^{1 / 2}(\Sigma)$.

Proof. We first simplify the notations we use. Let $\mu_{m}=\left(T_{\Sigma}\right)^{m} \mu$ and set $\left(\chi^{m}, \eta^{m+1}\right)=$ $\left(\chi_{\mu^{m}}, \eta_{\mu^{m}}\right)$. Therefore, we have $\mu_{m+1}=\left(\eta^{m+1}\right)_{\mid \Sigma}=\left(T_{\Sigma}\right)^{m+1} \mu=T_{\Sigma} \mu_{m}$. The convergence analysis will be conducted in the space $H^{1}\left(\Omega_{\gamma}\right)$. We need, therefore, to extend $\chi^{m} \in H^{1}\left(\omega_{\gamma}\right)$ by $\eta^{m}$, in $\Omega_{\gamma} \backslash \omega_{\gamma}$. The new function has no jump across $\Sigma$ and, hence, belongs to $H^{1}\left(\Omega_{\gamma}\right)$. By a notation abuse that functions is still denoted by $\chi^{m}$. We recall that $H^{1}(\Omega)$ is the Sobolev space on the safe domain (without cracks). The proof takes three steps. 
(i) Using equations (3.19)-(3.22) on $\eta^{m+1}$, where (3.20) reads as $\left[\eta^{m+1}\right]=\left[\chi^{m}\right]$, on $\gamma$ we deduce the variational relation

$$
\int_{\Omega_{\gamma}}\left(\eta^{m+1} \psi+c \nabla \eta^{m+1} \nabla \psi\right) d x=0, \quad \forall \psi \in H^{1}(\Omega) .
$$

Plugging $\chi^{m}$, it comes out that

$$
\begin{aligned}
& \int_{\Omega_{\gamma}}\left(\left(\eta^{m+1}-\chi^{m}\right) \psi+c \nabla\left(\eta^{m+1}-\chi^{m}\right) \nabla \psi\right) d x \\
= & -\int_{\Omega_{\gamma}}\left(\chi^{m} \psi+c \nabla \chi^{m} \nabla \psi\right) d x, \quad \forall \psi \in H^{1}(\Omega) .
\end{aligned}
$$

Since $\left[\chi^{m}-\eta^{m+1}\right]_{\mid \gamma}=0$, then $\left(\chi^{m}-\eta^{m+1}\right)$ belongs to $H^{1}(\Omega)$ and it is therefore the orthogonal projection of $\chi^{m}$ on $H^{1}(\Omega)$ with respect to the norm $\|\cdot\|_{H^{1}\left(\Omega_{\gamma}\right)}$ defined in (2.9). Consequently we obtain the Pythagore formula

$$
\left\|\chi^{m}-\eta^{m+1}\right\|_{H^{1}(\Omega)}^{2}+\left\|\eta^{m+1}\right\|_{H^{1}\left(\Omega_{\gamma}\right)}^{2}=\left\|\chi^{m}\right\|_{H^{1}\left(\Omega_{\gamma}\right)}^{2} .
$$

(ii) Writing down the variational formulation of (3.23)-(3.25) on $\chi^{m+1}$, after changing (3.24) into $\chi^{m+1}=\eta^{m+1}$ on $\Sigma$, requires to work with the closed convex set

$$
K_{\zeta, \eta^{m+1}}\left(\omega_{\gamma}\right)=\left\{\psi \in H^{1}\left(\omega_{\gamma}\right), \quad([\psi])_{\mid \gamma} \geq \zeta, \quad \psi_{\mid \Sigma}=\eta^{m+1}\right\} .
$$

Obviously $\chi^{m+1}$ belongs to $K_{\zeta, \eta^{m+1}}$ (so does $\eta^{m+1}$ ) and satisfies the variational inequality

$$
\int_{\omega_{\gamma}}\left(\left(\chi^{m+1}\left(\psi-\chi^{m+1}\right)+c \nabla \chi_{m+1} \nabla\left(\psi-\chi_{m+1}\right)\right) d x \geq 0, \quad \forall \psi \in K_{\zeta, \eta^{m+1}}\left(\omega_{\gamma}\right) .\right.
$$

Choosing $\psi=\left(\eta^{m+1}\right)_{\mid \omega_{\gamma}} \in K_{\zeta, \eta^{m+1}}$, we obtain that

$$
\int_{\omega_{\gamma}}\left(\left(\chi^{m+1}\left(\eta^{m+1}-\chi^{m+1}\right)+c \nabla \chi_{m+1} \nabla\left(\eta^{m+1}-\chi_{m+1}\right)\right) d x \geq 0\right.
$$

Owing to the formula $2(a, b-a)=-a^{2}-(a-b)^{2}+b^{2}$ we derive that

$$
\left\|\chi^{m+1}-\eta^{m+1}\right\|_{H^{1}\left(\omega_{\gamma}\right)}^{2}+\left\|\chi^{m+1}\right\|_{H^{1}\left(\omega_{\gamma}\right)}^{2} \leq\left\|\eta^{m+1}\right\|_{H^{1}\left(\omega_{\gamma}\right)}^{2} .
$$

Recalling that $\chi^{m+1}=\eta^{m+1}$ outside $\omega_{\gamma}$, we come up with the estimate on the whole domain $\Omega_{\gamma}$

$$
\left\|\chi^{m+1}-\eta^{m+1}\right\|_{H^{1}\left(\Omega_{\gamma}\right)}^{2}+\left\|\chi^{m+1}\right\|_{H^{1}\left(\Omega_{\gamma}\right)}^{2} \leq\left\|\eta^{m+1}\right\|_{H^{1}\left(\Omega_{\gamma}\right)}^{2} .
$$


(iii) Summing up both formulas (5.1) and (5.2) yields that

$$
\sum_{k=0}^{m}\left\|\chi^{k+1}-\eta^{k+1}\right\|_{H^{1}\left(\Omega_{\gamma}\right)}^{2}+\sum_{k=0}^{m}\left\|\chi^{k}-\eta^{k+1}\right\|_{H^{1}\left(\Omega_{\gamma}\right)}^{2}+\left\|\chi^{m+1}\right\|_{H^{1}\left(\Omega_{\gamma}\right)}^{2} \leq\left\|\chi^{0}\right\|_{H^{1}\left(\Omega_{\gamma}\right)}^{2} .
$$

We deduce first that $\left(\chi^{m}\right)_{m}$ is bounded in $H^{1}\left(\Omega_{\gamma}\right)$. Since that $\mu^{m}=\left(\chi^{m}\right)_{\mid \Sigma}$, then using the trace theorem yields that $\left(\mu^{m}\right)_{m}$ is also bounded in $H^{1 / 2}(\Sigma)$. The second result is that $\left(\left\|\chi^{m+1}-\eta^{m+1}\right\|_{H^{1}\left(\Omega_{\gamma}\right)}\right)_{m}$ and $\left(\left\|\chi^{m}-\eta^{m+1}\right\|_{H^{1}\left(\Omega_{\gamma}\right)}\right)_{m}$ are both decaying toward zero and so does $\left(\left\|\chi^{m+1}-\chi^{m}\right\|_{H^{1}\left(\Omega_{\gamma}\right)}\right)_{m}$ by the triangular inequality. Another use of the trace theorem produces that $\left(\mu^{m+1}-\mu^{m}\right)_{m}$ converges toward zero. $T_{\Sigma}$ is then asymptotically regular. The proof is complete.

\subsection{Convergence Result}

Aggregating the results of Lemmas 5.1 and 5.2, together with the abstract convergence of Proposition 4.1, we are able to state the final convergence of the Total Overlapping Schwarz algorithm.

Proposition 5.1. The sequences $\left(\eta^{m}\right)_{m}$ and $\left(\chi^{m}\right)_{m}$ computed by the total overlapping Schwarz method (3.8)-(3.11) and (3.12)-(3.14) converges towards $\varphi$ and $\varphi_{\mid \omega_{\gamma}}$ where $\varphi$ is the exact solution of the variational inequality (2.10),

$$
\lim _{m \rightarrow \infty}\left\|\varphi-\chi^{m}\right\|_{H^{1}\left(\omega_{\gamma}\right)}+\left\|\varphi-\eta^{m}\right\|_{H^{1}\left(\Omega_{\gamma}\right)}=0 .
$$

Proof. The result is proven is we check that the Picard sequence $\left(\lambda_{m}\right)$ computed in (3.27) converges towards the fixed point $\lambda$ of $T_{\Sigma}$ in $H^{1 / 2}(\Sigma)$. Since $T_{\Sigma}$ is continuous compact and asymptotically regular and that $\left(\lambda_{m}\right)_{m}$ is bounded we deduce that it is convergent toward $\lambda$. The proof is then complete.

Remark 5.1. Proposition 5.1 tells that the convergence of the Schwarz algorithm is guaranteed for $\Sigma$ arbitrarily chosen provided that $\Sigma \cap \gamma=\varnothing$. The evaluation of the convergence rate remains an open issue. We believe that, similarly to the linear cracks, it should be linear. This is related to the hypothetic contractivity of the operator $T_{\Sigma}$. Additionally, that convergence speed should be dependant on the size of the overlapping region and is expected to be higher for thicker $\omega_{\gamma}$.

\section{Conclusion}

The extension of the TOSM for the numerical simulation of Signorini unilateral cracks is the purpose of the present. We describe how the alterning Schwarz' approach is used as a zooming in procedure for the computation of variational solution at the vicinity of cracks with unilateral contact conditions. The convergence analysis based on Picard's sequence provide the proofs of the reliability of this method. Implementing this Substructuring method in the FreeFem code, developed by F. Hecht ([21]), is underway. 


\section{References}

[1] R. A. Adams. Sobolev Spaces. Academic Press, New York, (1975).

[2] S. Amdouni, K. Mansouri, Y. Renard and et al. Numerical convergence and stability of mixed formulation with X-FEM cut-off. Eur. J. Comput Mech., 21, 160-173, 2012.

[3] J. B. Apoung-Kamga and O. Pironneau. Numerical zoom and application to nuclear waste. J. Comput. Phys., 224: 403-413, 2007.

[4] L Badea and J. Wang. An additive Schwarz method for variational inequalities. Math. Comput., 69: 1341-1354, 2000.

[5] T. Belytschko, R. Gracie and G. Ventura. A review of extended/generalized finite element methods for material modeling. Model. Simul. Mater. Sci. Eng., 17, 2009.

[6] F. Ben Belgacem, M. Fournié, N. Gmati and tt al. On the Schwarz algorithms for the elliptic exterior boundary value problems. ESAIM, Math. Model. Numer. Anal., 39: 693-714, 2005.

[7] H. Ben Dhia, J. Olivier. On the use of XFEM within the Arlequin framework for the simulation of crack propagation. Comput. Meth. Appl. Mech. Eng., 199, 1403-1414, 2010.

[8] F. Brezzi, J.-L. Lions and O. Pironneau. On the chimera method. C. R. Acad. Sci. Paris, (I) Math. 332, 655-660, 2001.

[9] E. Chahine. Etude mathématique et numérique de méthodes d'éléments finis étendues pour le calcul en domaines fissurés. PhD., Institut National des Sciences Appliqués de Toulouse, 2008.

[10] E. Chahine, P. Laborde and Y. Renard. Crack-tip enrichment in the Xfem method using a cut-off function. Int. J. Numer. Meth. Engng., 75, 629-646, 2008.

[11] M. Ipopa and T. Sassi. A Robin domain decomposition algorithm for contact problems: convergence results. Lecture Notes in Computational Science and Engineering. M. Bercovier, M. J. Gander, R. Kornhuber, O. Widlund. Domain Decomposition Methods in Science and Engineering XVIII, 145-152, 2009.

[12] G. Duvaut and J.-L. Lions. Les inéquations en mécanique et en physique, Dunod, Paris, 1972.

[13] B.L. Karihaloo and Q.Z. Xiao. Modelling of stationary and growing cracks in FE framework without remeshing: a state-of-the-art review. Comput. Struct., 81: 119-129, 2003.

[14] F. Liu and R I. Borja. Finite deformation formulation for embedded frictional crack with the extended finite element method. Int. J. Numer. Meth. Eng., 82, 773-804, 2010.

[15] R. Glowinski, J. He, J. Rappaz and et al. A multi-domain method for solving numerically multi-scale elliptic problems. C. R. M. 338: 741-746, 2004.

[16] R. Glowinski, J. He, A. Lozinski and et al. Finite element methods with Patches and applications. DDM in Science and Engineering XVI. Lecture Notes in Computational Science and Engineering, 55: 77-, 2007,.

[17] P. Grisvard. Boundary value problems in non-smooth domains. Monographs and Studies in Mathematics, Pitman, London, 24, 1985.

[18] P.-A. Guidault, O. Allix, L. Champaney and et al. A multiscale extended finite element method for crack propagation. Comput. Meth. Appl. Mech. Engin., 197: 381-399, 2008.

[19] J. Haslinger, I. Hlaváček and J. Nečas. Numerical methods for unilateral problems in solid mechanics, in Handbook of Numerical Analysis, Volume IV, Part 2, Eds. P.G. Ciarlet and J.-L. Lions, North Holland, 1996.

[20] J. He, A. Lozinski and J. Rappaz. Accelerating the method of finite element patches using approximately harmonic functions. C. R. Acad. Sci. Paris (I) Math., 345: 107-112, 2007.

[21] F. Hecht. FreeFem++, User's Guide, 2011. Université Pierre et Marie Curie, Paris. On the web at http://www.freefem.org/ff++/ftp/freefem++doc.pdf.

[22] F. Hecht, A. Lozinski, and O. Pironneau. Numerical zoom and the Schwarz algorithm, Finite 
Element Methods with Patches and Applications, DDM in Science and Engineering XVIII, Lecture Notes in Computational Science and Engineering, 70: 63-74, 2009.

[23] F. Jelassi. Sur les méthodes de Schwarz pour les problèmes extérieurs. Application au calcul des courants de Foucault en électrotechnique. Thèse de l'Université Paul Sabatier, Toulouse III, France, 2006.

[24] P. Kerfriden, O. Allix and P. Gosselet. A three-scale domain decomposition method for the 3D analysis of debonding in laminates. Comput. Mech., 44: 343-362, 2009.

[25] N. Kikuchi and J. T. Oden. Contact Problems in Elasticity: A Study of Variational Inequalities and Finite Element Methods, SIAM, 1988.

[26] P. Ladeveze, A. Nouy, O. Loiseau. A multiscale computational approach for contact problems. Comput. Meth. Appl. Mech. Engin., 191: 4869-4891, 2002.

[27] P.-L. Lions. On the alternating Schwarz method I. In R. Gowinski, G. H. Golub, G. A. Meurant, J. Périaux, editors. First International Symposium on Domain Decomposition Methods for Partial Differential Equations, SIAM, Philadelphia, 1-42, 1988.

[28] W. K. Liu, S. Haox, T. Belytschko and et al. Multi-scale methods. Int. J. Numer. Meth. Engng., 47: 1343-1361, 2000.

[29] A. Lozinski. Méthodes numériques et modélisation pour certains problèmes multi-échelles HDR. Université Paul Sabatier, 2010.

[30] B. Maury. A fat boundary method for the Poisson equation in a domain with holes. J. Sci. Comput., 16: 319-339, 2001.

[31] P. Ming and X. Yue. Numerical methods for multiscale elliptic problems. J. Comput. Physic, 214: 421-445, 2006.

[32] N. Moës, N. Sukumar, B. Moran and et al. An extended finite element method (X-FEM) for two- and three-dimensional crack modeling, in ECCOMAS 2000, Barcelona, Spain, September, 11-14, 2000.

[33] N. Moës, J. Dolbow and T. Belytschko. A finite element method for crack growth without remeshing. Int. J. Numer. Meth. Engng., 46: 131-150, 1999.

[34] O. Pironneau. Numerical Zoom for localized Multi-Scale Problems. Mafelap, 2009.

[35] R. Ribeaucourt, M.-C. Baietto Dubourg and A. Gravouil. A new fatigue frictional contac$\mathrm{t}$ crack propagation model with the coupled XFEM/LATIN method. Comput. Meth. Appl. Mech. Engin., 196: 3230-3247, 2007.

[36] G. Strang and G. Fix. An Analysis of the Finite Element Method. Prentice-Hall, Englewood Clis, 1973.

[37] N. Sukumar, N. Moës, B. Moran and et al. Extended finite element method for three dimensional crack modelling. Intern. J. Numer. Meth. Engin., 48: 1549-1570, 2000.

[38] V. Visseque. Calcul haute performance en dynamique des contacts via deux familles de décomposition de domaine. PhD. Université de Montpellier, 2, 2013.

[39] E. Wyart, M. Duflot, D. Coulonand et al. Substructuring FE-XFE approaches applied to threedimensional crack propagation. J. Comput. Appl.Math., 215: 626-638, 2008. 